\section{Epidemiological distribution of rodents as potent reservoirs for infectious diseases in the provinces of Mazandaran, Gilan and Golestan, northern Iran}

\author{
Behzad Esfandiari, ${ }^{1}$ \\ Hossein Nahrevanian, ${ }^{2}$ \\ Mohammad Reza Pourshafie, ${ }^{3}$ \\ Mohammad Mehdi Gouya, ${ }^{4}$ \\ Pejvak Khaki, ${ }^{5}$ Ehsan Mostafavi, ${ }^{1}$ \\ Jamshid Darvish, ${ }^{6}$ Hamed Hanifi ${ }^{1}$ \\ ${ }^{1}$ Department of Epidemiology and \\ Biostatistics, ${ }^{2}$ Department of \\ Parasitology, ${ }^{3}$ Department of \\ Bacteriology, Pasteur Institute of Iran, \\ Tehran; ${ }^{4}$ Centre for Diseases Control \\ and Prevention, Ministry of Health, \\ Tehran; ${ }^{5}$ Department of Microbiology, \\ Razi Vaccine and Serum Research \\ Institute, Agricultural Research, \\ Education and Extension Organization \\ (AREEO), Karaj; ' ${ }^{6}$ Department of \\ Biology, Ferdowsi University \\ of Mashhad, Iran
}

\begin{abstract}
Rodents are mammals that comprise more than 2000 species and approximately 30 families. There are many morphological and ecological differences among them as variations in their shape, size, weight and habitat. In addition to significant economic losses, rodents have a major role in the dissemination of infectious diseases caused by viruses, bacteria, parasites or other micro-organisms Rodents are important reservoirs of diseases which have been observed in many cities of Iran provinces especially along Caspian Sea border to Alborz Mountain. The aim of this study is to assess the geographical distribution of rodents in three provinces of northern part of Iran as reservoir of potential endemic infectious diseases. Rodents in 10 major parts of each of the three provinces of Mazandaran, Gilan and Golestan, northern Iran were collected and a total of 404 rodents were trapped alive. They were determined by the key characteristics such as gender, genus, species, different locations and topological situation. Statistical analysis was performed to characterize the study sample and to correlate all variables and parameters. The distribution frequencies of three, five and six genera of rodents were identified in Mazandaran, Gilan and Golestan provinces respectively. The overall distribution frequency of eight genera
\end{abstract}

of rodents in the three provinces were identified as Rattus (R.) norvegicus (67.3\%), R. rattus (13.6\%), Apodemus sylvaticus (13.9\%), Arvicola (1\%), Mus musculus (0.3\%), Nesokia indica (2.5\%), Cricetulus migrates $(0.7 \%)$ and Rhombomys opimus $(0.7 \%)$. The results of this study determined the geographic distribution of the rodents in the three northern provinces of Iran. It is indicated the association of various distribution and diversity of rodents with provincial location. The overall distribution frequency of eight genera of rodents was recognized in the above three provinces geographical locations. This study confirms epidemiological distribution of various rodents as potent reservoirs for infectious diseases, such as leptospirosis, salmonellosis, tularemia, leishmaniasis, etc. in the three provinces.

\section{Introduction}

Rodents are mammals of the order Rodentia. Order of rodents comprises more than 2000 species and approximately 30 families. ${ }^{1}$ There are many biological and ecological differences among rodents. Many variations are observed in shape, size and weight of them; whereas the smallest rodents (mice), weighing 5 grams and the largest rodent, weighing more than $70 \mathrm{~kg} .{ }^{1}$ Some species live above the trees while others live underground and are rarely out of the ground. Some of the rodents are dependent on the water and they live behind the water, while others are accustomed to living in dry and desert areas. The different behaviors observed among various rodents, particularly various and different motor behaviors and characteristics such as jumping, climbing, swimming, running, fast movements, digging and moving underground. Also, there are different systems in rodents in terms of social life. Rodents are able to eat a variety of foods, including animal and plants such as roots, stems, bark, leaves, fruits and seeds of plants, insects and small creatures, and even rodent droppings and other animals. They play a special role in the chain in natural communities; they are considered as food for many species of predators and on the other hand, by feeding on small creatures such as insects and arthropods, they control their populations. ${ }^{1,2}$ Rats by feeding on crops, livestock, stored grains and foods can cause loss of economic resources and also by chewing wires and telephone and damaging to electrical appliances, they expose installations and houses to great risks. ${ }^{3,4}$ Rodents can do damage to products in a wide range, before and after harvest. In addition to significant economic losses, rodents have a major role in the dissemination of infectious diseases. It is
Correspondence: Hossein Nahrevanian, Department of Parasitology, Pasteur Institute of Iran, Tehran, Iran.

Tel./Fax: +98.21.66968855.

E-mail: mobcghn@gmail.com

Key words: Rodents; Iran; Infectious Disease; Reservoir; Mazandaran; Gilan; Golestan.

Acknowledgments: this study was supported by Pasteur Institute of Iran, in collaboration with the Microbiology Department, Razi Vaccine and Serum Research Institute, Karaj, Iran and the Centre for Diseases Control and Prevention, Ministry of Health, Tehran, Iran. Results presented in this article are part of a $\mathrm{PhD}$ thesis (Behzad Esfandiari) from the Pasteur Institute of Iran, Tehran, Iran under the second and third authors' supervision.

Contributions: HN, MRP, study concept and design; $\mathrm{BE}, \mathrm{HH}$, field sampling; EM, analysis and interpretation of data; MMG, field management; PK, laboratory diagnosis; JD, rodentology. All authors read and approved the final manuscript.

Conflict of interest: the authors declare no potential conflict of interest.

Funding: this work was entirely supported by the Pasteur Institute of Iran, (Project No. 739).

Received for publication: 22 September 2016. Revision received: 10 November 2016. Accepted for publication: 30 January 2017.

This work is licensed under a Creative Commons Attribution-NonCommercial 4.0 International License (CC BY-NC 4.0).

(C) Copyright B. Esfandiari et al., 2017 Licensee PAGEPress, Italy

Infectious Disease Reports 2017; 9:6900 doi:10.4081/idr.2017.6900

estimated that each year, approximately 20 percent of the world's food supply is consumed or damaged by rodents. ${ }^{5,6}$ According to the World Health Organization (WHO) report, about 3 million tons of food (5\% of the total global food production) annually destroyed by mice that will be adequate to feed 130 million hungry people. ${ }^{7}$

Diseases carried by rodents include viral, bacterial, parasitic infections. ${ }^{5,8}$ The disease can be transmitted to humans in a number of ways including animal bite, contact with animal waste, eating food or water contaminated by rodent waste or through parasites that use rodents and humans as hosts e.g. fleas and ticks. ${ }^{9,10}$

Rats are potential important reservoirs of infectious disease from human in Iran. ${ }^{3}$ Due to the spread of these diseases there is 
a strengthen fight against rats. ${ }^{11}$ Iran, lying on the crossroad of several biogeographic regions, supports a diverse mammal fauna. More than 79 rodent species have been recorded from Iran (38.2\% of species). ${ }^{12}$ Three species of the genus Rattus Fisher, 1803 have been reported from Iran: $R$. norvegicus (brown rat, Berkenhout, 1769), $R$. rattus (black rat, Linnaeus, 1758) and $R$. pyctoris (Himalayan rat, Hodgson, 1845) The first two species of Rattus genre have a worldwide distribution. The brown rat has also moved from central Asia to the border of the Caspian Sea and to Gorgan and Rasht cities in the north. ${ }^{13}$ There are numerous cytogenetic studies about Black rats. These studies indicate the value of diversity chromosomes within this genus. ${ }^{14}$ Rodents are reported to be almost one third of the species of mammals in Iran. ${ }^{1,2}$ The temperate and humid climate ranges from the plains along Caspian Sea border to northern foothills of Alborz Mountainin Iran. In this region, rice planting is the predominant occupation and the most peasants are farmers and keep one or more livestock in their houses. In mentioned region, surface waters or rivers are mainly used for agriculture. ${ }^{3}$ The aim of this paper is to assess the geographical and epidemiological distribution of various rodents as potential reservoirs and vector for human infectious diseases in the three provinces of Mazandaran, Gilan and Golestan, northern Iran (Middle East Asian region).

\section{Materials and Methods}

\section{Study area and sample collection}

This study was performed during the summer of 2013 close to Caspian sea (Latitude $41^{\circ} \mathrm{N}$ and Longitude $50^{\circ} \mathrm{E}$; the average altitude above sea level is 1305 $\mathrm{m} / 4281 \mathrm{ft}$ ) in three northern provinces of Iran, Mazandaran Province (Sari, Nour and Noshahr districts), Gilan Province (Roodsar, Langerood, Lahijan, Rasht, Fooman, Some'esara, Masouleh, Bandar Anzali, Talysh and Astara) and Golestan Province (Gorgan, Kordkuy, Kohneh, Azadshahr, MinooDasht, Kalaleh, GonbadKavoos, Maraveh Tappeh, Incheh Boroun and Bandar Torkaman). In this study, active colonies of rodents in 10 major parts of each of the three provinces and a total of 404 rodents were trapped alive (Figure 1). They were determined and categorized by the key characteristics such as gender, genus, species, different locations, topological situation and morphological characters including the shape and size of tail, claw, fur, nose, vibrissa, digit and pinna.

aimed to correlate all statistical variables and parameters.

\section{Data analysis}

Statistical analysis was performed in two stages using SPSS version 17. The first was a descriptive which aimed to characterize the study sample. The second stage was

\section{Results}

The distribution frequency of three genera of rodents identified in Mazandaran

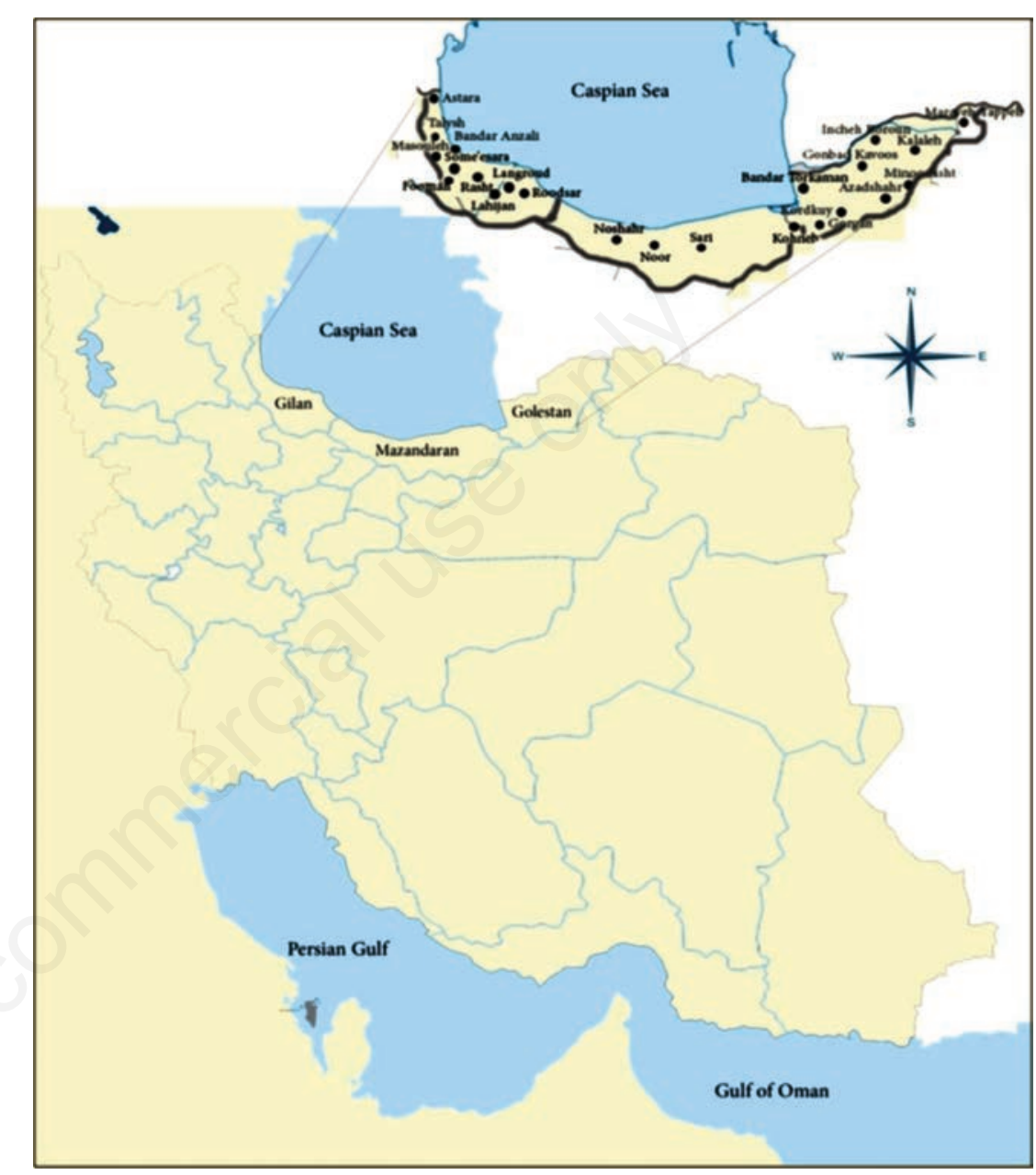

Figure 1. Map of study area in the three provinces of Mazandaran, Gilan and Golestan, northern Iran.

Table 1. The genus and number of the rodents in three northern provinces of Iran.

\begin{tabular}{lcccc} 
Rodent species & Mazandaran & Gilan & Golestan & Total \\
Rattus norvegicus & 116 & 102 & 54 & 272 \\
Rattus rattus & 9 & 32 & 14 & 55 \\
\hline Apodemus sylvaticus & 26 & 12 & 18 & 56 \\
Arvicola spp. & 0 & 4 & 0 & 4 \\
\hline Mus musculus & 0 & 1 & 0 & 1 \\
Nesokia indica & 0 & 0 & 10 & 10 \\
\hline Cricetulus migratus & 0 & 0 & 3 & 3 \\
Rhombomys opimus & 0 & 0 & 3 & 3 \\
\hline Total & 151 & 151 & 102 & 404
\end{tabular}


Province were Rattus (R.) norvegicus (76.8\%), R. rattus (6.0\%) and Apodemus (A.) sylvaticus $(17.2 \%)$. Moreover, five genera of rodents were recognized in Gilan Province as $R$. norvegicus $(67.5 \%), R$. rattus $(21.2 \%)$, A. sylvaticus (7.9\%), Arvicola spp. $(2.6 \%)$ and Mus musculus $(0.7 \%)$ and finally six genera of rodents were reported in Golestan Province as $R$. norvegicus (54\%), R. rattus $(13.7 \%)$, A. sylvaticus (17.6\%), Nesokia indica (9.8\%), Cricetulus migrates $(2.9 \%)$ and Rhombomys opiimus $(2.9 \%)$ (Table 1$)$.

The overall distribution frequency of eight genera of rodents in the three provinces of Mazandaran, Gilan and Golestan were identified as $R$. norvegicus (67.3\%), R. rattus (13.6\%), Apodemus sylvaticus (13.9\%), Arvicola (1\%), Mus musculus $(0.3 \%)$, Nesokia indica (2.5\%), Cricetulus migrates $(0.7 \%)$ and Rhombomys opimus $(0.7 \%)$ (Table 1$)$.

\section{Discussion and Conclusions}

The results of this study determined the geographic distribution of the rodents in the three Northern provinces of Iran. It is indicated the association of various distribution and diversity of rodents with provincial location. The distribution frequencies of three, five and six genera of rodents were identified in Mazandaran, Gilan and Golestan provinces respectively. The overall distribution frequency of eight genera of rodents was recognized in the above three provinces geographical locations. The major rodents were reported as $R$. norvegicus $(67.3 \%)$, R. rattus (13.6\%) and A. sylvaticus $(13.9 \%)$. These data is supported by previous report indicated more than 79 rodent species have been recorded from Iran earlier. ${ }^{12}$ Three species of the genus Rattus have been reported from Iran: $R$. norvegicus, $R$. rattus and $R$. pyctoris. The brown rat has also moved from central Asia to the border of the Caspian Sea and to Gorgan and Rasht cities in the north. ${ }^{13}$

According to Centers for Diseases Control and Prevention (CDC), there are several diseases directly transmitted by rodents including Hemorrhagic fever with Renal Syndrome, Lassa fever, Leptospirosis, Lymphocytic Chorio-meningitis (LCM), Omsk Hemorrhagic Fever, Rat-Bite Fever, Salmonellosis, South American Arenaviruses (Argentine hemorrhagic fever, Bolivian hemorrhagic fever, Sabiá-associated hemorrhagic fever, Venezuelan hemorrhagic fever) and Tularemia. In addition, several diseases are also indirectly transmitted by rodents with biological vectors including Colorado Tick Fever, Leishmaniasis, Anaplasmosis, La Crosse Encephalitis, Lyme Disease, Murine Typhus, Omsk Hemorrhagic Fever, Powassan Virus, Scrub Typhus, Rickettsial pox, Relapsing Fever, Rocky Mountain Spotted Fever, Sylvatic Typhus, Tularemia, Plague and West Nile Encephalitis (see Appendix). ${ }^{9,15-17}$

There are several reports indicating the potential important role of synanthropic rodents as key reservoirs of several infectious diseases in Iran. According to Wikipedia, 2017 the major rodents Genera reported in Iran are Hystrix, Sciurus, Spermophilopsis, Spermophilus, Castor, Dryomys, Myomimus, Glis, Allactaga, Pygeretmus, Dipus, Jaculus, Spalax, Calomyscus, Cricetulus, Mesocricetus, Arvicola, Blanfordimys, Ellobius, Microtus, Acomys, Gerbillus, Meriones, Rhombomys, Apodemus, Golunda, Mus, Nesokia and Rattus. The main infectious diseases were transmitted by rodents reported to be Leptospirosis, Tularemia, Plague, Leishmaniasis, leprosy, Tuberculosis, Ratbite fever, Cryptosporidiosis, Echinococcosis and several Ectoparasites, Endoparasitic protozoa and helminthes. Major infectious diseases which are transmitted by rodents have been almost observed in almost entire provinces of Iran. ${ }^{18-21}$ Therefore, the issue in the fight against rodents has become more important than past. Iran, lying on the crossroad of several bio-geographic regions, supports a diverse mammal fauna. Due to the wide variety of climate and abundance of different species of domestic and wild rodents in Iran, consistent study is essential to monitor the status of density, mobility and variety of rodents population. Identification of habitats and faunistic composition of these animals should always be considered as a tool to control the rodents. Awareness of local people about the harmful effects of rodents is recommended for public health education. Moreover, local health authorities should identify the major points of presence of rodents and activate specific control programs through the use of anticoagulant baits.

\section{References}

1. Kay EH, Hoekstra HE. Rodents. Curr Biol 2008;18:R406-10.

2. Kumawat M, Singh K, Sen D, et al. Threatened rodent species of Arunachal Pradesh. Int J Agricult Envir Biotechnol 2013;6:657-68.

3. Esfandiari B, Pourshafie MR, Gouya $\mathrm{MM}$, et al. An epidemiological compa- rative study on diagnosis of rodent leptospirosis in Mazandaran Province, northern Iran. Epidemiol Health 2015;37:e2015012.

4. Tobin ME, Fall MW. Pest control: rodents. USDA National Wildlife Research Center-Staff Publications. 2004:67.

5. Meerburg BG, Singleton GR, Kijlstra A. Rodent-borne diseases and their risks for public health. Critic Rev Microbiol 2009;35:221-70.

6. Ehrlich PR, Ehrlich AH, Daily GC. Food security, population and environment. Pop Develop Rev 1993:1-32.

7. Von der Weid D, Dillon J, Falquet J. Malnutrition: a silent massacre. Geneva: Antenna Technologies; 2000.

8. Waugh CA, Lindo JF, Foronda P, et al. Population distribution and zoonotic potential of gastrointestinal helminths of wild rats Rattus rattus and $R$. norvegicus from Jamaica. J Parasitol 2006;92:1014-8.

9. Bitam I, Dittmar K, Parola P, et al. Fleas and flea-borne diseases. J Infect Dis 2010;14:e667-76.

10. Han BA, Schmidt JP, Bowden SE, et al. Rodent reservoirs of future zoonotic diseases. Proc Natl Acad Sci USA 2015;112:7039-44.

11. Pourmohammadi B, Motazedian M, Kalantari M. Rodent infection with Leishmania in a new focus of human cutaneous leishmaniasis, in northern Iran. Ann Trop Med Parasitol 2008;102:127-33.

12. Karami M, Hutterer R, Benda P, et al. Annotated check-list of the mammals of Iran. Lynx (Praha) 2008;39:63-102.

13. Jalal R, Darvish J, Moghaddam Matin M. Identification of three Iranian species of the genus Rattus (Rodentia, Muridae) using a PCR-RFLP technique on mitochondrial DNA. Hystrix-Ita J Mammal 2009;20.

14. Steen RG, Kwitek-Black AE, Glenn C, et al. A high-density integrated genetic linkage and radiation hybrid map of the laboratory rat. Genom Res 1999;9:AP18.

15. Centers for Diseases control and Prevention. Diseases from rodents. 2015. Available from: http://www.cdc.gov/rodents/diseases/in direct.html.

16. Helhazar M, Leitão J, Duarte A, et al. Natural infection of synathropic rodent species Mus musculus and Rattus norvegicus by Leishmania infantum in Sesimbra and Sintra-Portugal. Parasit Vector 2013;6:88.

17. Bonnefoy X, Kampen H, Sweeney et al. Public health significance of urban 
pests. Copenhagen: World Health Organization-Regional Office for Europe; 2008. 569 pp

18. Nateghpour M, Motevalli-Haghi A, Akbarzadeh K, et al. Endoparasites of Wild Rodents in Southeastern Iran._J Arthropod Borne Dis 2014;9:1-6.

19. Mowlavi G, Makki M, Mobedi I, et al. Paleoparasitological Findings from
Rodent Coprolites Dated At 500 CE Sassanid Era in Archeological Site of Chehrabad (Douzlakh), Salt Mine Northwestern Iran. Iran J Parasitol 2014;9:188-93.

20. Meshkekar M, Sadraei J, Mahmoodzadeh A, et al. Helminth Infections in Rattus ratus and Rattus norvigicus in Tehran, Iran. Iran J
Parasitol 2014;9:548-52.

21. Yousefi A, Eslami A, Mobedi I, et al. Helminth infections of house mouse (Mus musulus) and wood mouse (Apodemus sylvaticus) from the Suburban Areas of Hamadan City, Western Iran. Iran J Parasitol 2014;9:511-8. 